\title{
COUNTABLE UNIONS OF TOTALLY PROJECTIVE GROUPS(1)
}

\author{
BY
}

\author{
PAUL HILL
}

\begin{abstract}
Let the $p$-primary abelian group $G$ be the set-theoretic union of a countable collection of isotype subgroups $H_{n}$ of countable length. We prove that if $H_{n}$ is totally projective for each $n$, then $G$ must be totally projective. In particular, an ascending sequence of isotype and totally projective subgroups of countable length leads to a totally projective group. The result generalizes and complements a number of theorems appearing in various articles in the recent literature. Several applications of the main result are presented.
\end{abstract}

In [6], the author proved that if a $p$-primary abelian group $G$ is the set-theoretical union of a countable number of pure subgroups $H_{n}$, then $G$ is a direct sum of cyclic groups if $H_{n}$ is for each $n$. In the present paper, we generalize this result by proving that if the subgroups $H_{n}$ are isotype then $G$ must be totally projective provided that $H_{n}$ is totally projective of countable length for each $n$. Although we do not resolve the case where the subgroups $H_{n}$ are allowed to have arbitrary length, the restriction to countable length is enough to unify a number of recent theorems from [1], [6], [7], [9], [10] and [11]. This theorem also provides the basis for some additional new results. We remark that even if the theorem is valid for groups of length $\Omega$, few, if any, applications should be expected for this case because such groups tend not to be countable set-theoretic unions of significantly simpler isotype and totally projective subgroups.

For terminology, as well as for the fundamental techniques, the reader is referred to [2, Chapter V] or to original sources such as [5] and [7]. In particular, we shall assume the equivalence of being totally projective and satisfying the author's third axiom of countability. It will be tacitly assumed throughout that all groups are $p$-primary abelian groups.

Although it is not truly essential, we employ the following lemma in the proof of our main theorem.

Lemma 1. Let $G$ be a p-primary group of countable length and let

Received by the editors August 23, 1972.

AMS (MOS) subject classifications (1970). Primary 20K10; Secondary 20K25.

Key words and phrases. Abelian p-groups, totally projective groups, isotype subgroups, ascending chain, Kulikov's criterion.

(1) This research was supported, in part, by NSF grant GP-29025. 


$$
0=A_{0} \subseteq A_{1} \subseteq A_{2} \subseteq \cdots \subseteq A_{\alpha} \subseteq \cdots, \quad a<\mu,
$$

be a cbain (indexed by an arbitrary ordinal $\mu$ ) of nice subgroups of $G$ satisfying the following conditions:

(i) $A_{a+1} / A_{a}$ is countable.

(ii) $A_{\beta}=\bigcup_{a<\beta} A_{a}$ when $\beta$ is a limit.

(iii) $G=\bigcup_{a<\mu} A_{a}$.

Then $G$ is totally projective.

Proof. If $\mu \leq \Omega$, then it follows at once that $G$ satisfies the third axiom of countability. However, for an arbitrary $\mu$, it is not immediately evident that conditions (i)-(iii) imply the third axiom of countability.

Nevertheless, under the hypothesis, we can embed (by extending inductively from $A_{a}$ to $A_{a+1}$ a height-preserving monomorphism into a sufficiently large totally projective group) the group $G$ as an isotype subgroup of a totally projective group. Since the length of $G$ is countable, $G$ itself is totally projective by our theorem in [3].

Theorem 1. Suppose that the p-primary abelian group $G$ is the set-theoretical union of a countable number of isotype subgroups $H_{n}$. If $H_{n}$ is totally projective of countable length for each $n$, then $G$ is totally projective.

Proof. First, observe that $G$ must have countable length. Let the length of $G$ be $\lambda$ and let the subgroups $H_{n}$ be indexed by the nonnegative integers. For each $n<\omega$, let $H_{n}=\Sigma_{i \in I(n)} C_{i}$ where $C_{i}$ is countable for each $i$. Suppose that we have developed a chain

$$
0=A_{0} \subseteq A_{1} \subseteq A_{2} \subseteq \cdots \subseteq A_{a} \subseteq \cdots, \quad a<\tau,
$$

of subgroups of $G$ satisfying the following conditions:

(1) $A_{a+1} / A_{a}$ is countable.

(2) $A_{\beta}=\bigcup_{a<\beta} A_{a}$ when $\beta$ is a limit.

(3) $A_{a} \cap H_{n}=\Sigma_{i \in I(n, a)} C_{i}$ for each $n$ and $\alpha$, where $I(n, \alpha)$ is a subset of $I(n)$.

(4) $\left\langle p^{\gamma} G, A_{a}\right\rangle \cap\left\langle H_{n}, A_{a}\right\rangle=\left\langle p^{\gamma} H_{n}, A_{a}\right\rangle$ for each $n$ and $\alpha$ and for each $\gamma \leq \lambda$.

If $\tau$ is a limit ordinal, we define $A_{\tau}=\bigcup_{a<\tau} A_{a}$ and observe that conditions (1)-(4) continue to hold for the extended chain of subgroups $A_{a}$. Now suppose that $\tau-1$ exists. For an arbitrary countable extension $C$ of $A_{\tau-1}$ in $G$, there exists a countable extension $K$ of $C$ such that $K \cap H_{n}=\Sigma_{i \in J(n)} C_{i}$, for each $n$, where $J(n)$ is a countable extension of $I(n, \tau-1)$; we assume the reader is familiar with the back-and-forth procedure involved here (if this is not the case, see, for example, [2, Chapter V]).

We shall now give the essence of the argument that there exists a countable extension $B$ of $K \supseteq C \supseteq A_{\tau-1}$ in $G$ such that 


$$
\left\langle p^{\gamma} G, B\right\rangle \cap\left\langle H_{n}, B\right\rangle=\left\langle p^{\gamma} H_{n}, B\right\rangle
$$

for all $\gamma \leq \lambda$ and all $n<\omega$. Let $\left\{k_{i}\right\}_{i<\omega}$ be a set of representatives for the cosets of $K / A_{\tau-1}$. For each triple $(i, n, \gamma)$ with $i, n<\omega$ and $\gamma \leq \lambda$ such that $z+b_{n} \epsilon$ $k_{i}+A_{\tau-1}$ with $z \in p^{\gamma} G$ and $b_{n} \in H_{n}$, we choose a single representative $z=z_{i, n}, \gamma$ for the triple $(i, n, \gamma)$. Thus there are only a countable number of such representatives. Letting $B_{1}=\left\langle K, z_{i, n}, \gamma\right\rangle$, we observe that

$$
\left\langle p^{\gamma} G, K\right\rangle \cap\left\langle H_{n}, K\right\rangle \subseteq\left\langle p^{\gamma} H_{n}, B_{1}\right\rangle \text {. }
$$

If we let $B_{1}$ replace $K$ and then in turn let $B_{j+1}$ replace $B_{j}$ where $B_{j+1}=$ $\left\langle B_{j}, z_{i, n, \gamma}\right\rangle$, we see that $B=\bigcup_{j<\omega} B_{j}$ has the desired properties.

By altemately considering conditions (3) and (4), we obtain in $G$ a countable extension $A_{\tau}$ of $A_{\tau-1}$ containing $C$ that satisfies both conditions (3) and (4). Hence a chain satisfying conditions (1)-(4) can be developed until $G$ is exhausted. We shall show that $A_{a}$ is necessarily nice in $G$ for each $a$. It suffices to show

$$
p^{\gamma}\left(G / A_{\alpha}\right)=\left\langle p^{\gamma} G, A_{\alpha}\right\rangle / A_{a}
$$

for all $\gamma \leq \lambda$, which will be proved by induction on $\gamma$ in conjunction with the condition

$$
p^{\gamma}\left(G / A_{\alpha}\right) \cap\left\langle H_{n}, A_{a}\right\rangle / A_{a}=\left\langle p^{\gamma} H_{n}, A_{a}\right\rangle / A_{a}=p^{\gamma}\left(\left\langle H_{n}, A_{a}\right\rangle / A_{a}\right) .
$$

Obviously, for a given $\gamma$ the second equality in condition (II) is a consequence of the first. However, the second equality is valid, independent of the first, because of condition (3). Therefore, we observe that condition (II) complements condition (I) extremely well inasmuch as (II) automatically survives limit ordinals whereas (I) automatically survives isolated ordinals. Thus if (I) should fail, it must fail for the first time at a limit ordinal $\delta$. We claim that (II) must hold for $\gamma=\delta$. Since $\delta$ is a limit, it suffices to prove that (II) holds for all $\gamma<\delta$. By the choice of $\delta$, condition (I) holds for all $\gamma<\delta$. Hence, if $\gamma<\delta$, we have that

$$
p^{\gamma}\left(G / A_{\alpha}\right) \cap\left\langle H_{n}, A_{\alpha}\right\rangle / A_{\alpha}=\left(\left\langle p^{\gamma} G, A_{\alpha}\right\rangle \cap\left\langle H_{n}, A_{\alpha}\right\rangle\right) / A_{\alpha},
$$

but by condition (4) the latter is equal to $\left\langle p^{\gamma} H_{n}, A_{a}\right\rangle / A_{a}$. Thus condition (II) holds for $\gamma=\delta$. This implies that

$$
p^{\delta}\left(G / A_{a}\right) \subseteq \bigcup_{n<\omega}\left\langle p^{\delta} H_{n}, A_{a}\right\rangle / A_{\alpha} \subseteq\left\langle p^{\delta} G, A_{a}\right\rangle / A_{\alpha}
$$

which proves that $A_{a}$ is nice in $G$ for each $a$. The proof of the theorem is now a consequence of Lemma 1.

Remark. In the proof of Theorem 1, Lemma 1 could be omitted by directly 
exhibiting the validity of the third axiom of countability. However, since the proof of Lemma 1 is so short, we prefer to use it.

It was first proved in [7, Theorem 3.10] that if $G$ is a summable $p$-primary group of countable length $\lambda$, then $G$ is totally projective provided that $G / p{ }^{a} G$ is totally projective for each $\alpha<\lambda$. Later, C. Megibben [10, Theorem A] gave a different and more detailed proof of this theorem and referred to the result as "the generalized Kulikov criterion". We shall exhibit some of the strength of Theorem 1 by showing that it quickly yields the proof of the generalization of Kulikov's criterion just mentioned. At the same time, a very small (and purely artificial) improvement is effected.

Theorem 2. Suppose that $G$ is a summable p-primary group of countable length $\lambda$. Then $G$ is totally projective provided that $G / p^{a} G$ is totally projective for eacb limit ordinal $a<\lambda$.

Proof. The proof is by induction on $\lambda$. Since $G$ is totally projective if both $p^{a} G$ and $G / p^{a} G$ are, we may assume that $\lambda$ is a limit ordinal. Let $\lambda(1)<\lambda(2)<$ $\ldots<\lambda(n)<\ldots$ be an increasing sequence of ordinals whose limit is $\lambda$. Choose $H_{1} \subseteq H_{2} \subseteq \cdots \subseteq H_{n} \subseteq \cdots$ to be an increasing sequence so that $H_{n}$ is $p^{\lambda(n)_{\text {-high }}}$ in $G$ and so that $H[p]=G[p]$, where $H=\bigcup_{n<\omega} H_{n}$. Since $H$ is pure, $H=G$. Thus, in view of Theorem 1 , it suffices to prove that $H_{n}$ is totally projective for each $n$. However, we know that $H_{n}$ is isomorphic to an isotype subgroup of $G / p^{\lambda(n)} G$ under the natural map. Since the latter is totally projective, $H_{n}$ is too [3].

We shall now generalize Theorem 2 in [6], which states that if the p-primary group $G$ has cardinality $X_{a}$ where $\alpha$ is cofinal with $\omega$, then $G$ is a direct sum of cyclic groups provided each subgroup of $G$ having cardinality less that $\boldsymbol{N}_{a}$ is a direct sum of cyclic groups.

Theorem 3. Let $G$ be a p-primary abelian group of cardinality $K_{a}$ wbere $a$ is cofinal with $\omega$. If eacb subgroup of $G$ baving cardinality less than $\boldsymbol{K}_{a}$ is contained in a totally projective subgroup of $G$ baving countable length, then $G$ is necessarily totally projective.

Proof. Suppose that $H_{n}$ is a subgroup of $G$ having cardinality $\boldsymbol{X}_{a(n)}$, where $a(1)<a(2)<\ldots<a(n)<\cdots$ is an increasing sequence of ordinals whose limit is a. According to [4], $H_{n}$ is contained in an isotype subgroup $K_{n}$ of $G$ having the same cardinality as that of $H_{n}$, which is $K_{a(n)}$. By hypothesis, $K_{n}$ is contained in a totally projective subgroup $G_{n}$ of $G$ having countable length. Since $K_{n}$ is isotype in $G_{n}, K_{n}$ is also totally projective. It follows that $G$ is the union of a countable ascending sequence $K_{1} \subseteq K_{2} \subseteq \cdots \subseteq K_{n} \subseteq \cdots$ of isotype and totally projective subgroups $K_{n}$. By Theorem $1 G$ is totally projective.

One can also apply Theorem 1 to show that certain subgroups of totally 
projective groups are again totally projective. A paper by F. F. Kamalov [9] establishes the following. If $G$ is totally projective of length less than $\omega^{2}$ and if $H$ is a subgroup of $G$ such that $p^{a} G / p^{a} H$ is countable for all $a$, then $H$ is also totally projective. A far-reaching generalization of Kamalov's result has been provided by B. L. Edington [1]. As we have indicated, Theorem 1 can be used to establish results of this kind. Details in this connection will appear elsewhere.

As was pointed out in [10, Lemma 6.1], if $A$ and $B$ are $p^{\alpha}$-high subgroups of $G$ and if $\alpha=\beta+n$ where $\beta$ is a limit and $n<\omega$, then there exists a heightpreserving isomorphism between $\left(A / p^{\beta} A\right)[p]$ and $\left(B / p^{\beta} B\right)[p]$. More generally, it is not difficult to show that there exists a height-preserving isomorphism between $\left(A / p^{\beta} A\right)[p]$ and $\left(B / p^{\beta} B\right)[p]$ for every limit ordinal $\beta$.

R. Nunke [12] and C. Megibben [10] have given independent proofs that all $p^{a}$-high subgroups of $G$ are direct sums of countable groups if one $p^{a}$-high subgroup of $G$ is a direct sum of countable groups. Since the theorem is trivially true (in view of the results of [7]) if $\alpha=\Omega$, there is no loss of generality in assuming that $a$ is countable. Hence, in the light of the preceding remarks, this result is encompassed in the next theorem.

Theorem 4. Let $H$ and $K$ be p-groups of countable type $r$ and suppose that $H$ is totally projective. If, for eacb ordinal $a \leq r$, there exists a beight-preserving isomorphism between $\left(H / p^{\omega a} H\right)[p]$ and $\left(K / p^{\omega a} K\right)[p]$, then $K$ is totally projective and $H \cong K$.

Proof. By hypothesis, there exists a height-preserving isomorphism between $H[p]$ and $K[p]$. Thus the groups $H$ and $K$ have the same Ulm invariants, and the two are isomorphic if $K$ is totally projective.

We shall prove that $K$ is totally projective by induction on $r$. Since $H$ is summable, $K$ must be summable. If $r=1$, then $K$ is a direct sum of cyclic groups. Thus we may assume that $r>1$. Next, assume that $r-1$ exists. For simplicity of notation, let $G^{a}$ denote $p^{\omega a} G$. We observe that

$$
H / H^{\tau-1} /\left(H / H^{\tau-1}\right)^{a} \simeq H / H^{a}
$$

for all $a \leq \tau-1$, and likewise, of course, for $K$. By the induction hypothesis, $K / K^{\tau-1}$ is totally projective. Moreover, $K^{\tau-1}$ is a direct sum of cyclic groups since $K^{\tau-1}[p]$ and $H^{\tau-1}[p]$ have a height-preserving isomorphism between them. Therefore $K$ is totally projective. Now we consider the case that $r$ is a limit ordinal. Let $H[p]=\Sigma S_{a}$ and $K[p]=\Sigma T_{a}$ be decompositions of $H[p]$ and $K[p]$, respectively, such that the nonzero elements of $S_{a}$ and $T_{a}$, respectively, have height exactly $a$. Letting $1 \leq \pi(1)<r(2)<\ldots<r(n)<\ldots$ be an increasing sequence of ordinals whose limit is $r$, we choose ascending sequences 
$A_{1} \subseteq A_{2} \subseteq \cdots \subseteq A_{n} \subseteq \cdots$ and $B_{1} \subseteq B_{2} \subseteq \cdots \subseteq B_{n} \subseteq \cdots$ where $A_{n}$ and $B_{n}$, respectively, are chosen maximal with respect to $A_{n}[p]=\Sigma_{a<\omega \tau(n)} S_{a}$ and $B_{n}[p]=$ $\Sigma_{a<\omega \tau(n)} T_{a}$. Note that $H=\bigcup_{n<\omega} A_{n}$ and $K=\bigcup_{n<\omega} B_{n}$ since $\bigcup_{n<\omega} A_{n}$ and $\bigcup_{n<\omega} B_{n}$ are pure subgroups of $H$ and $K$, respectively, containing $H[p]$ and $K[p]$.

We wish to show that $B_{n}$ is totally projective and our plan is to use the induction hypothesis of the present theorem. Thus we need to show the existence of a height-preserving isomorphism between $\left(A_{n} / A_{n}^{a}\right)[p]$ and $\left(B_{n} / B_{n}^{a}\right)[p]$ for each $\alpha \leq \pi(n)$. In order to do so we need to consider two cases. If $\alpha=\pi(n)$, then there is a height-preserving isomorphism between $\left(A_{n} / A_{n}^{a}\right)[p]=A_{n}[p]$ and $\left(B_{n} / B_{n}^{a}\right)[p]=$ $B_{n}[p]$ by the choice of $A_{n}$ and $B_{n}$. Thus it suffices to consider the case that $a<\gamma(n)$. Recall that $H=\left\langle A_{n}, H^{a}\right\rangle$ since $a<\gamma(n)$ and since $A_{n}$ is $p^{\omega \tau(n)_{\text {-high }}}$ in $H$. Similarly, $K=\left\langle B_{n}, K^{a}\right\rangle$. Therefore,

$$
H / H^{a} \cong A_{n} / A_{n}^{a} \text { and } K / K^{a} \cong B_{n} / B_{n}^{a} .
$$

We conclude that there exists a height-preserving isomorphism between $\left(A_{n} / A_{n}^{a}\right)[p]$ and $\left(B_{n} / B_{n}^{a}\right)[p]$ if $a<\pi(n)$. By the induction hypothesis, $B_{n}$ is totally projective. Since $B_{n}$ is isotype in $K$ and since $K=\bigcup_{n<\omega} B_{n}$, Theorem 1 implies that $K$ is totally projective.

We should remark that an alternate proof of Theorem 4 could be obtained from [10, Theorem 6.7].

Although it is not our purpose to do so here, the so-called $C_{\lambda}$-theory initiated by Hill and Megibben [8] and later developed more fully by Megibben [11] could be established now using Theorem 1 to great advantage. In fact a restricted technical version of Theorem 1 (concerning $\lambda$-high towers) was an essential requirement in the original development of the $C_{\lambda}$-theory [11].

\section{REFERENCES}

1. B. L. Edington, Isomorphic invariants in quotient categories of abelian groups, Dissertation, New Mexico State University, 1971.

2. Phillip Griffith, Infinite abelian group theory, Univ. of Chicago Press, Chicago, III., 1970. MR 44 \#6826.

3. Paul Hill, Isotype subgroups of direct sums of countable groups, Illinois J. Math. 13 (1969), 281-290. MR 39 \#1550.

4. - The purification of subgroups of abelian groups, Duke Math. J. 37 (1970), 523-527. MR 42 \#365.

5. - On the classification of abelian groups, Xeroxed notes, 1967.

6. - Primary groups whose subgroups of smaller cardinality are direct sums of cyclic groups, Pacific J. Math. 41 (1972) 63-67.

7. P. Hill and $C$. Megibben, $O$ n direct sums of countable groups and generalizations, Studies on Abelian Groups (Sympos., Montpellier, 1967), Springer, Berlin, 1968, pp. 183206. MR 39 \# 4270. 
8. P. Hill and C. Megibben, $O_{n}$ certain classes of primary abelian groups, Notices Amer. Math. Soc. 15 (1968), 105. Abstract \#653-93.

9. F. F. Kamalov, The subgroups of direct sums of countable abelian groups, Vestnik Moskov. Univ. Ser. I Mat. Meh. 26 (1971), no. 1, 31-35. (Russian) MR 43. \#6307.

10. C. Megibben, The generalized Kulikov criterion, Canad. J. Math. 21 (1969), 1192-1205. MR 40 \#2754.

11. _ A generalization of the classical theory of primary groups, Tôhoku Math. J. (2) 22 (1970), 347-356. MR 45 \#3561.

12. R. Nunke, On the structure of Tor. II, Pacific J. Math. 22 (1967), 453-464. MR 35 \# 5508.

DEPARTMENT OF MATHEMATICS, FLORIDA STATE UNIVERSITY, TALLAHASSEE, FLORIDA 32306 